\title{
Purification of CD34+ Stem Cells from Myelodysplastic Syndrome with Ring Sideroblasts using Immunomagnetic Strategy
} \author{
Drénou $^{1,2}$ \\ ${ }^{1}$ Institut de Recherche en Hématologie et Transplantation (IRHT), Mulhouse, France \\ ${ }^{2}$ Groupement Hospitalier Régional Mulhouse Sud Alsace (GHRMSA), Mulhouse, France \\ ${ }^{3}$ Cellprothera, Mulhouse, France \\ *Corresponding author: Romain Barbet, IRHT, Mulhouse, France
}

Romain Barbet*1, Antoinette Eidenschenk ${ }^{1}$, Ines Harzallah ${ }^{2}$, Philippe Hénon ${ }^{1,3}$ and Bernard

\section{ARTICLE INFO}

Received: 蔧 March 11, 2021

Published: 㹃 March 29, 2021

Citation: Romain Barbet, Antoinette Eidenschenk, Ines Harzallah, Philippe Hénon, Bernard Drénou. Purification of CD34+ Stem Cells from Myelodysplastic Syndrome with Ring Sideroblasts using Immunomagnetic Strategy. Biomed J Sci \& Tech Res 34(5)-2021. BJSTR. MS.ID.005613.

Keywords: Myelodysplastic Syndrome; Ring Sideroblasts; CD34; Immunomagnetic Column; Purification

\section{ABSTRACT}

Background and Aim: While CD34+ stem cell purification can be easily achieved for most samples using magnetic activated cell sorting, it is a clue when working on diseases such as Myelodysplastic Syndromes (MDS) especially for the Ring Sideroblastic (RS) subcategories. High iron content of cells characterizing these patients induces a much lower purity due to contaminant cells.

Methods: Herein, we developed an efficient and robust procedure to isolate highly purified CD34+ cells from MDS-RS using magnetic column. Flow cytometry analysis demonstrates the presence of erythroid and monocyte derived cells in samples purified from MDS-RS.

Results: Precleaning column did magnetically retain these cells leading to a better purification percentage of the hematopoietic stem cells. Results have been confirmed by MGG and Perls staining.

Conclusion: Malignant clonal cells purification is a clue when working on diseases such as Myelodysplastic Syndromes. We believe that these data may help for developing an alternative procedure for studying MDS-RS stem cells with a higher purity.

Abbreviations: MDS: Myelodysplastic Syndromes; RS: Ring Sideroblastic; HSC: Hematopoietic Stem Cell; BM: Bone Marrow; IMBs: Immunomagnetic-Beads; MNC: Mononuclear Cells; PcC: Precleaning Column; MF: Magnetic Fraction

\section{Introduction}

Myelodysplastic syndromes (MDS) are a heterogeneous group of hematopoietic disorders frequently leading to severe anemia. A subcategory of this disease involves the multiplication of mutated erythroid cells with iron overload. This ineffective hematopoiesis named MDS with Ring Sideroblasts (MDS-RS) is mainly related to spliceosome mutations, especially SF3B1 gene, leading to excess iron accumulation in mitochondria from the multipotent lymphomyeloid
Hematopoietic Stem Cell (HSC) compartment to the most mature erythroid cells. Research on MDS-RS may require the purification of the bone marrow hematopoietic stem cells (BM-HSC). This purification is mainly based on two basic approaches to separate highly purified population of HSC. First, flow cytometry cell sorting is an accurate technic leading to well purified cells. Nevertheless, it requires to be equipped with a cell sorter and to apply some rules for a good achievement [1]. Moreover, this approach could be time 
consuming for a large sample. The second approach using magnetic bead separation technology has the advantages to be simple, robust and automatable. This immunomagnetic-beads (IMBs) cell sorting approach is usually giving a high percentage of purity, above 9095\% pure populations, but could be variable depending on the sample origin, the disease or the model used. Indeed, using IMBs separation remain challenging for the MDS-RS disease as multiple labs reported a lower purity level than those traditionally obtained with this technic $[2,3]$.

We also observed this issue during our attempt to purified high-quality CD34+ cells from MDS-RS samples, unlike other MDS categories such as MDS with Excess Blasts (MDS-EB). In the particular case of the MDS-RS, this technic suffers from inherent limitations of magnetic-based cell separation. The Ring Sideroblasts characterization supposed that cells are so much overloaded with iron that these accumulations could be visualized as blue dot through a Perls staining. As Martin et al. demonstrated it to purify sideroblasts, a magnetic field can be used to collect magnet-positive cells in mouse [4] and human [5]. By this approach, the iron-loaded cells such as sideroblasts or siderocytes remained stick on the magnetic column due to their high level of magnetic metals. We hypothesized that it would be possible to purify a high percentage of HSC from MDS-RS using IMBs approach if the iron overloaded cells were first removed. Herein, we describe a simple and robust procedure for the purification of highly purified CD34+ HSC from human bone marrow samples with MDS-RS disease. By employing a magnetic column-based method followed by a classical magnetic bead-based selection, we were able to obtain a pure population of CD34+ HSC. Moreover, we provide evidence that this effective method for cell separation mainly retained erythroid cells which are highly positive for Perls staining.

\section{Materials and Methods}

\section{Sample Collection and Patients Characteristics}

Sternal bone marrow aspirations were obtained from 16 patients with MDS after informed consent during a diagnostic sampling and were preserved in a media containing heparin. This cohort include 8 patients ( 3 males, 5 females, median age of 69.5 years ranging from 52 to 88 years) with myelodysplastic syndrome with ring sideroblasts (MDS-RS), and 8 patients ( 6 males, 2 females, median $=73$ years, range $=52-86$ years $)$ with MDS with excess of blast (MDS-EB). Samples were classified according to WHO criteria [6]. In addition, bone marrow cells from 6 healthy controls ( 4 males, 2 females, median $=61$ years, range $=31-74$ years) were obtained during cardiac surgery after informed consent.

\section{Precleaning and Purification of CD34+ Cells}

Bone marrow mononuclear cells (MNC) were isolated by using lymphocytes separation medium (Eurobio, Courtaboeuf, France) density gradient according to standard procedures. For already diagnosed or suspected MDS-RS, a precleaning column (PcC) was added using the following procedure. Briefly, in absence of any magnetic bead affinity reagent, MNCs are passed through MACS Columns (Miltenyi Biotec, Paris, France) activated with the manufacturer's recommended buffer (PBS-BE) based on PBS (Eurobio, Courtaboeuf, France) containing 0.5\% bovine serum albumin (BSA; Sigma Aldrich, St Quentin Fallavier Cedex, France), and 2 mM EDTA (Sigma Aldrich). The choice of the column size is determined by the MNC number. After 2 washes with PBS-BE, while eluted cells in the magnetic fraction (MF) are set apart, not retained cells are then enriched for CD34+ cells using magnetic-activated cell sorting (MACS; Miltenyi Biotec) Direct CD34 Progenitor Cell Isolation Kit according to manufacturer's protocol.

\section{Multicolor Flow Cytometry Immunophenotyping for CD34+ Purity Determination}

Following separation, cells were suspended in $100 \mu \mathrm{L}$ of PBS and incubated with the following antibodies anti CD34-FITC (Becton Dickinson, Le Pont de Claix, France), anti CD235a-PE (Ozyme/Biolegend, St Quentin en Yvelines, France), anti CD71-APC (Miltenyi Biotec), anti CD45-APC-Vio770 (Miltenyi Biotec), anti CD36-VB (Miltenyi Biotec) and the nucleic acid Laser Dye Styryl 751 [7] (LDS-751, Molecular Probe/Invitrogen, Life technologies, Villebon sur Yvette, France) at 200ng/mL. After 15 minutes of incubation at room temperature, samples were washed once with $2 \mathrm{~mL}$ of PBS and centrifuged at $600 \mathrm{~g}$ for $10 \mathrm{~min}$. The supernatant was discarded, and the cells were resuspended in $100 \mu \mathrm{L}$ of PBS. Samples were analyzed onto a flow cytometer (FACS Canto II, BD) and FCS files exported for hematopoietic cells identification using Infinicyt software (Cytognos, Spain).

\section{Morphological and Iron Assessment}

Cells from different steps of purification were cytocentrifugated using Hettich Universal 16 (5 minutes, 45g) onto glass slides at $1 \times 10^{5}$ cells/slide in $100 \mu \mathrm{L}$ of PBS completed with $4 \%$ human albumin. After drying, slides were stored in the dark at room temperature or directly used for staining. The cells morphologies is determined by May-Grünewald Giemsa (MGG) staining realized by incubating slides in May-Grünewald solution for 2 minutes, then 3 minutes in Weise buffer and 5 minutes in 5\% Giemsa solution before an intensive final wash with water. Iron content is determined by Perls staining with classical procedure. Briefly, after 10 minutes of methanol fixation/permeabilization, the slides are dry and incubated with a mix solution of $2 \%$ ferrocyanic potassium and $0.2 \mathrm{~N} \mathrm{HCl} \mathrm{(v/v)} \mathrm{for} 30$ minutes. After intensive wash with water, cells are counterstain during 10 minutes with filtered Harris' hematoxylin solution (Papanicolaou's solution 1a) and again wash with water.

Cells are observed with an AxioStar Plus microscope (Zeiss, Germany). 


\section{Statistical Analysis}

After validation of the parametric distribution with KolmogorovSmirnoff test, paired or unpaired two-tailed Student t-tests were used to compare datasets. Comparison between MGG and Flow cytometry or clinical data and cells retained on precleaning column results were tested with Pearson correlation assay. P values $<0.05$ were considered significant.

\section{Results}

\section{CD34+ purity in MDS Patients}

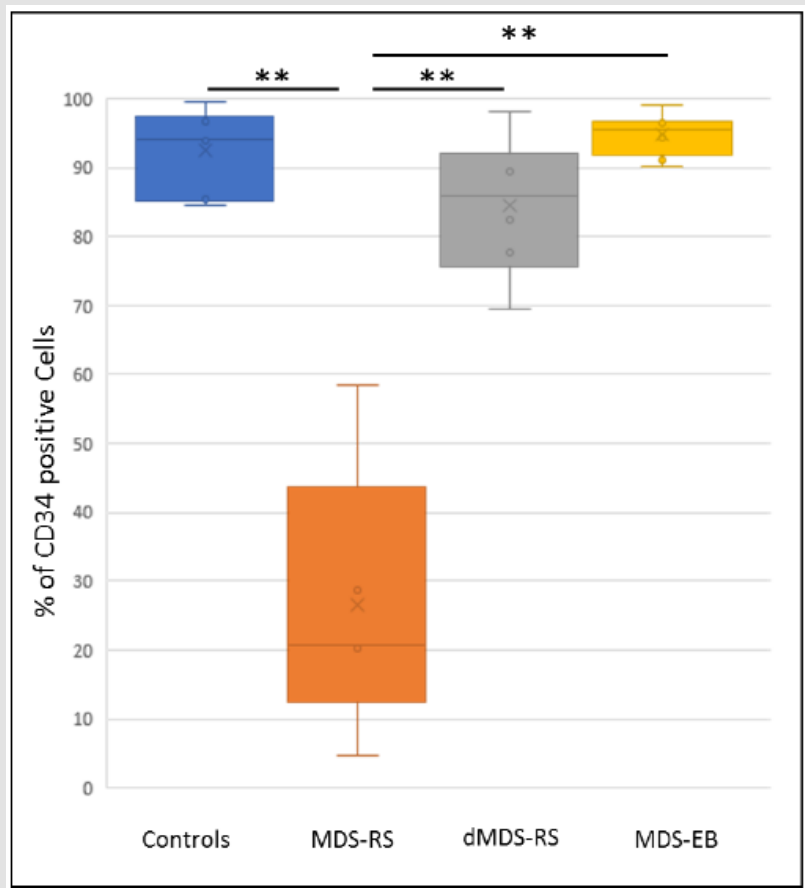

Figure 1: Purity of CD34+ cells determined by Flow cytometry after immunomagnetic beads-based purification. Controls, Myelodysplastic Syndrome with Ring Sideroblast (MDS-RS), MDS-RS previously depleted on a precleaning magnetic column (dMDS-RS) or MDS with Excess Blasts (MDS-EB) samples are analyzed. Percentages of CD34+ cells are lower in MDS-RS than in Controls, MDS-EB or dMDS-RS. ${ }^{* *}$ pValue $<0.01$ (Student $\mathrm{t}$ test).

After IMBs based purification, we determined the percentages of CD34+ cells using Flow Cytometry (FC) in the different samples. As summarized in (Figure 1), there was no difference $(p=0.456)$ in percentage of CD34+ cells purity between control group reaching a median of $94.1 \%$ (range $85 \%-99 \%$ ) and MDS-EB group with 95.5\% (range 90\%-97\%). While CD34+ cells for these 2 last groups were purified with high percentages, the MDS-RS samples purity was lowering to a median value of $20.7 \%$ with percentages ranging from $5 \%$ to $59 \%$. This significant differences between MDS-RS and control or MDS-EB ( $p=0.0012$ and $p=0.0015$ respectively) can be abolished by the addition of a precleaning column (PcC) for MDSRS samples. By this approach, CD34+ cells purification values from depleted MDS-RS (dMDS-RS) are significantly $(\mathrm{p}=0.0013)$ going back to a median value of $86 \%$ (range from $78 \%$ to $98 \%$ ) compared to the original MDS-RS. Moreover, comparisons between control or MDS-EB samples with dMDS-RS give nonsignificant results ( $>>0.05)$ which clearly confirm that this approach improve CD34+ purification close to control and MDS-EB groups.

\section{MDS-RS are retained on the Precleaning Column}

To determine the nature of the cells contaminating MDS-RS after CD34+ population's purification or magnetically stuck on the PcC, we tested the presence of different membrane markers into the retained cells including the CD235a (Glycophorin A) expressed by erythroid precursors and erythrocytes. Our gating strategies includes the selection of the interesting population according to their FSC/SSC profile and nucleated cell discrimination by staining nucleic acids with Laser Dye Styryl-751 [7] (LDS-751, data not shown). We observed that the majority of cells are CD235a+ in MDS-RS and PcC samples (Figure 2A events in red) compared to control samples, dMDS-RS or MDS-EB. Without PcC, FC results analysis demonstrate a mean of $65.8 \%(+/-11.6)$ of erythroid cells that are contaminating the MDS-RS CD34+ fraction (in dark blue) after purification (Figure 2B). At the same time, CD235a+ cells represent $81.7 \%(+/-5.5)$ of the retained fraction of the PcC. Interestingly, the MGG staining (Figures 3A-3E) for MDS-RS, PcC and Controls respectively and counts realized in parallel on the separated fractions confirm these results with a significant degree of correlation with FC analysis (data not shown, $\mathrm{R}^{2}=0.8, \mathrm{p}=0.016$ ). Indeed, we observe in (Figure 3) that control CD34+ represents a homogeneous population while MDS-RS and PcC eluted fractions contain contaminating cells.

Absence or low number of CD235a+ events (in red) in controls, dMDS-RS or MDS-EB is resulting into high percentage of CD34+ cells observed (dark blue in Figures 2A \& 2B). Unexpectedly, we observed that a small population of CD34+ cells could be identified after PcC. Even if this population is low $(1.0 \%+/-0.3)$ compared to the number of CD235a+ events, these cells may represent a part of the malignant clone. Finally, even if erythroid cells are the major contaminant population, we also identified a lower percentage of others cells including, from most to least abundant, immature granulocytes (IG, in green), monocytes/macrophages (in light blue) and lymphocytes (in orange) that were identified according to their FSC/CD45 profiles (Figure 2A). All these cells could be trapped by the magnetic column as illustrated in Figure 3. Since the iron overloaded erythroid population is the major contaminant observed during CD34 purification from MDS-RS bone marrow samples, these cells can be easily depleted using PcC. 

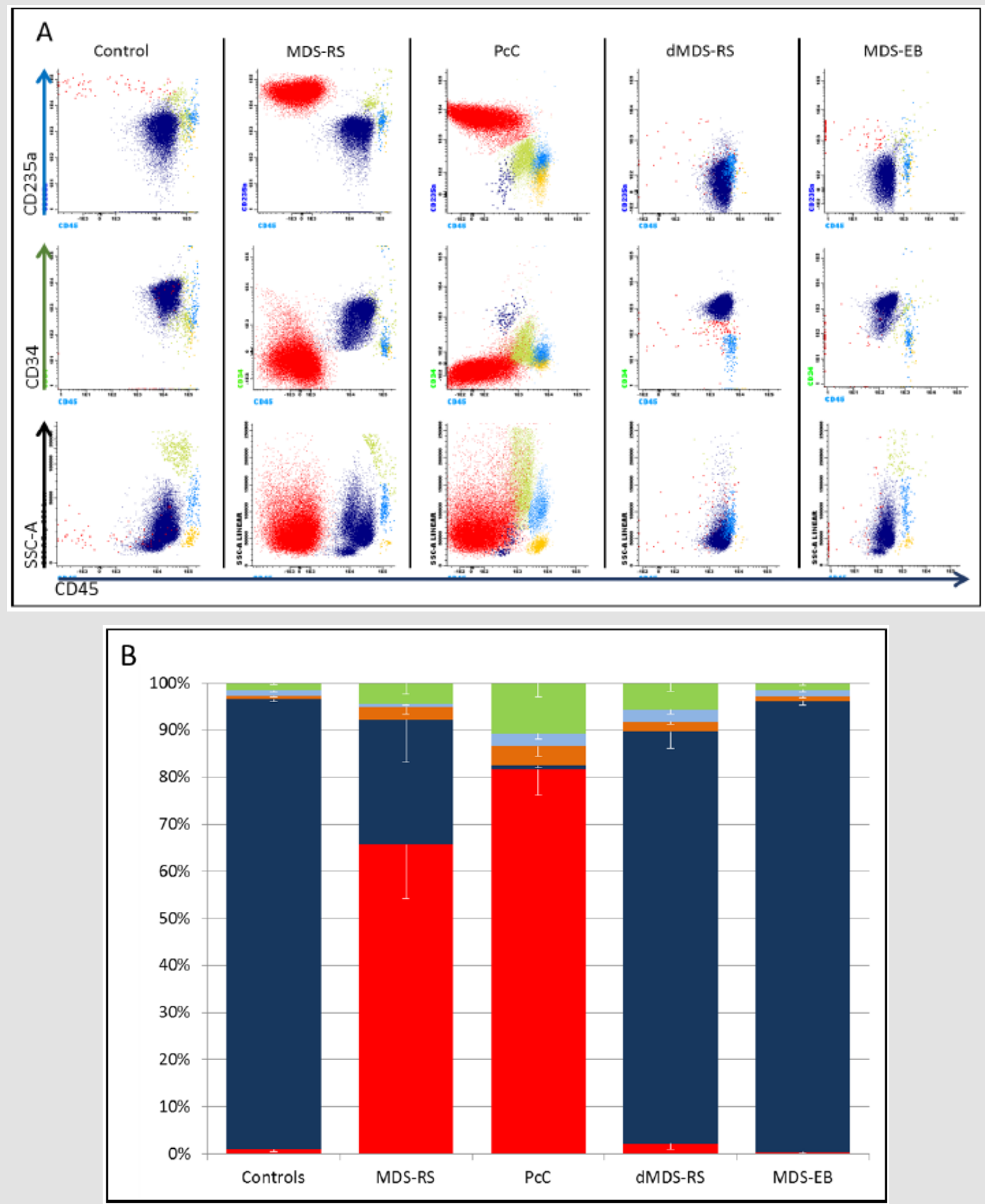

Figure 2: Cell populations in different samples using Flow cytometry.

A. Representative flow cytometry dot plot allowing characterization of CD34+ Hematopoietic Stem Cells (HSC, Dark Blue), erythroid cells (Red), lymphocytes (orange), monocytes/macrophages (light blue) and Immature Granulocytes (IG, green) in controls, MDS-RS, retained cells on Precleaning Column (PcC), dMDS-RS and MDS-EB. Nucleated cells firstly selected for their LDS-751 integration are further analyzed for their CD235a+/CD45- expression which represent the erythroid progenitor cells; CD34+/CD45+ events identifies the HSC population and finally, lymphocytes, monocytes/macrophages and IG are identified according to their CD45+/SSC profiles.

B. Flow cytometry data percentages are shown as mean values $(+/-\mathrm{SEM} ; \mathrm{n}=6)$ of the different cells and represented as follow: CD34+ population (dark blue), lymphocytes (orange), monocytes/macrophages (light blue) and granulocytes (green). The precleaning column retained particularly erythroid cells and monocyte derived cells. 


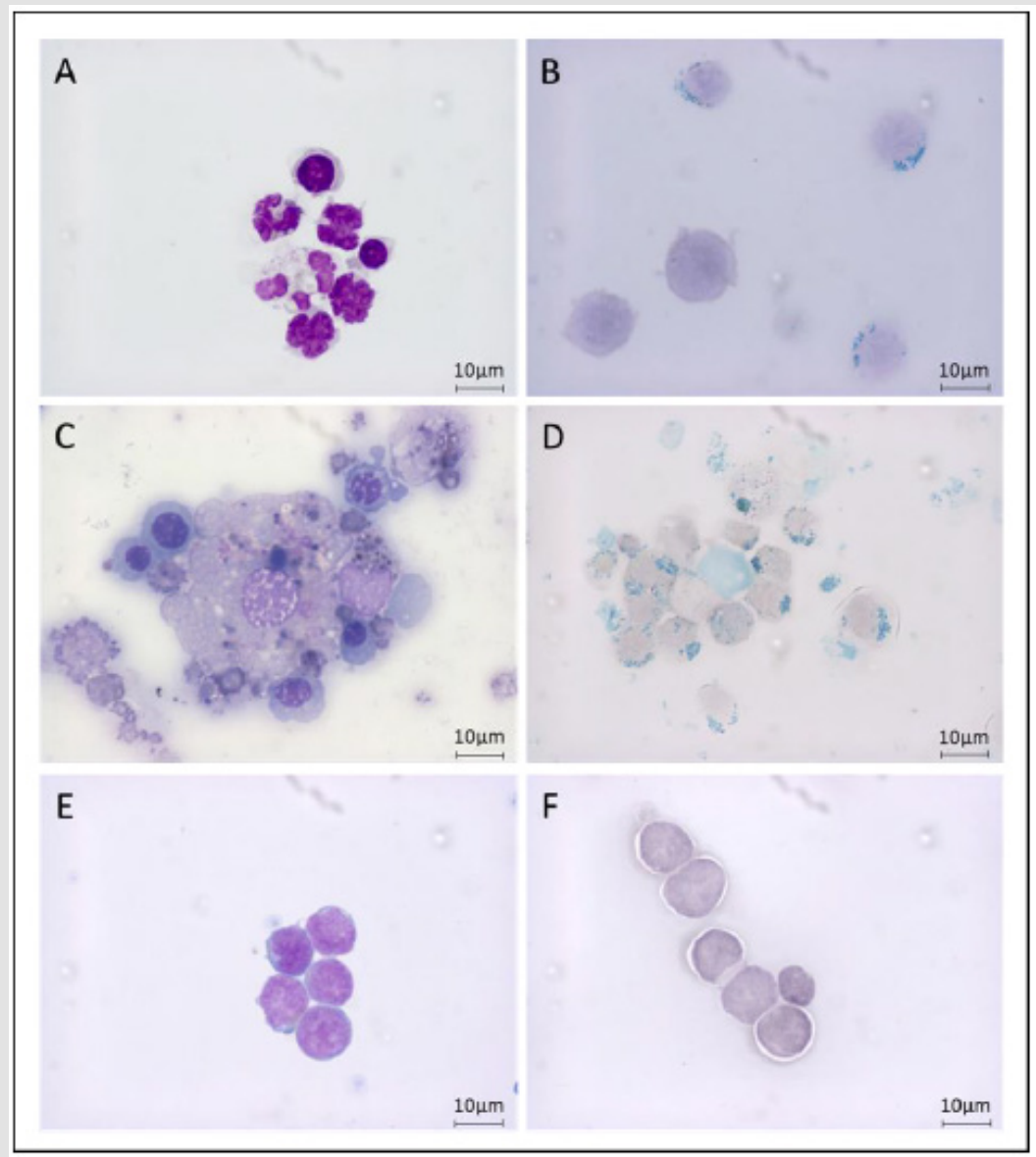

Figure 3: Cell fractions in MDS-RS bone marrow during purification process.

Morphological characterization of cells retained during CD34+ purification on MDS-RS (A and B), the precleaning column (C and D) or controls (E and F). Erythroid progenitor cells, immature granulocytes and monocytes derived cells morphology can be assessed on MGG coloration (A and C) and compared to CD34+ HSC from controls (E). Cells with iron overload (mainly sideroblasts and macrophages) were easily observed after Perls staining (B and D) while controls samples are negative (F). Scale bar $=10 \mu \mathrm{m}$.

\section{Clinical Comparison}

As demonstrated in (Figures 3B \& 3D), cells contaminating MDS-RS eluted fraction or retained on PcC were positive for Perls staining contrary to CD34+ from control samples (Figure 3F). We speculated that the percentage of cells retained on PcC might correlate with the quantity of RS on BM smears but no relationship between percentage of RS and percentage of cells retained on the columns was significantly observed in our small cohort (data not shown, $n=5, p=0.056$ ).

\section{Discussion}

Myelodysplastic syndromes are malignant diseases characterized by different hallmarks including ineffective erythropoiesis, severe anemia and iron accumulation for the MDS-RS subtype. Our experience on MDS-RS and previous studies from others described variable percentages of purified CD34+ cells using Immunomagnetic approach [2,3]. Herein, we provide evidence that purification of cells using IMBs-based approach for
MDS-RS samples leads to the contamination of unwanted cells with high iron overload including a large majority of erythroid cells. Our experiments also confirmed the results of Martin, et al. [4,5] about sideroblasts/siderocytes purification. Interestingly, if most cells retained on $\mathrm{PcC}$ were from the erythroblast compartment, we also purified granulocytes and monocytes/macrophages. The explanations for their purification could be a direct interaction with high iron content cells or the possibility that these cells contain themselves a high level of iron. As granulocytes and macrophages have phagocytic capabilities, it would not be surprising that their detection may be also related to their high iron content cell cleaning ability. Furthermore, macrophages are the bone marrow iron regulators and our approach probably selected the iron overloaded macrophages and discarded the others.

We also identified CD34+ cells among the retained cells. As MDS is a clonal disease, we may hypothesize that our approach also retained part of the abnormal progenitor proerythroblast population. Finally, comparison of Perls' prussian blue results 
realized on bone marrow smear with our FC data lead to a nonsignificant relationship. This difference could be due to the gradient separation method that induces siderocytes/sideroblasts loss [8] before the PcC step. To conclude, getting a high percentage of purified CD34+ cells with immunomagnetic methods on MDSRS is impossible without using a precleaning column. We provide evidence that cells retained on the column are mainly expressing CD235a marker and are identified as erythroid cells by MGG. We believe that these data obtained from a small cohort of patients may help in developing further alternative processes for studying MDSRS.

\section{Author Contributions}

a. RB, designed experiments, interpreted the data and drafted the work,

b. AE, acquired, analyzed, interpreted the experimental data and helped write the manuscript,

c. IH, acquired and analyzed the clinical data,

d. PH, revised critically the draft and add intellectual content,

e. BD, interpreted the data, revised critically the draft with important intellectual content, gave final approval of the publication.

f. All authors have read and approved the final version of the manuscript.

\section{Acknowledgement}

We would like to thank the cardiac surgeons, Dr Bischoff and Dr Gavra, for providing control samples and patients giving their authorization for the present work.

ISSN: 2574-1241

DOI: 10.26717/BJSTR.2021.34.005613

Romain Barbet. Biomed J Sci \& Tech Res

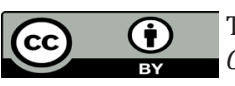

This work is licensed under Creative

Commons Attribution 4.0 License

Submission Link: https://biomedres.us/submit-manuscript.php

\section{Funding Sources}

This work was supported by the departmental council of HautRhin.

\section{Disclosure of Potential Conflicts of Interest}

The authors indicate no potential conflicts of interest.

\section{References}

1. Cossarizza A, Chang HD, Radbruch A, Andreas Acs, Dieter Adam, et al. (2017) Guidelines for the use of flow cytometry and cell sorting in immunological studies. Eur J Immunol 47(10): 1584-1797.

2. Nilsson L, Astrand Grundström I, Anderson K, Ingrid Arvidsson, Peter Hokland, et al. (2002) Involvement and functional impairment of the CD34(+)CD38(-)Thy-1(+) hematopoietic stem cell pool in myelodysplastic syndromes with trisomy 8. Blood 100(1): 259-267.

3. Elvarsdóttir EM, Mortera Blanco T, Dimitriou M, Thibault Bouderlique, Monika Jansson, et al. (2020) A three-dimensional in vitro model of erythropoiesis recapitulates erythroid failure in myelodysplastic syndromes. Leukemia 34(1): 271-282.

4. Martin FM, Bydlon G, Welsh ML, Friedman JS (2005) A method for rapid mouse siderocyte enrichment. Exp Hematol 33(12): 1493-1499.

5. Martin FM, Prchal J, Nieva J, Alan Saven, Jeffrey Andrey, et al. (2008) Purification and characterization of sideroblasts from patients with acquired and hereditary sideroblastic anaemia. British Journal of Haematology 143(3): 446-450.

6. Arber DA, Orazi A, Hasserjian R, Jürgen Thiele, Michael J Borowitz, et al. (2016) The 2016 revision to the World Health Organization classification of myeloid neoplasms and acute leukemia. Blood 127(20): 2391-2405.

7. Wiczling P, Krzyzanski W (2008) Flow cytometric assessment of homeostatic aging of reticulocytes in rats. Exp Hematol 36(2): 119-127.

8. Pösel C, Möller K, Fröhlich W, Schulz I, Boltze J, et al. (2012) Density Gradient Centrifugation Compromises Bone Marrow Mononuclear Cell Yield. PLOS ONE 7(12): e50293.

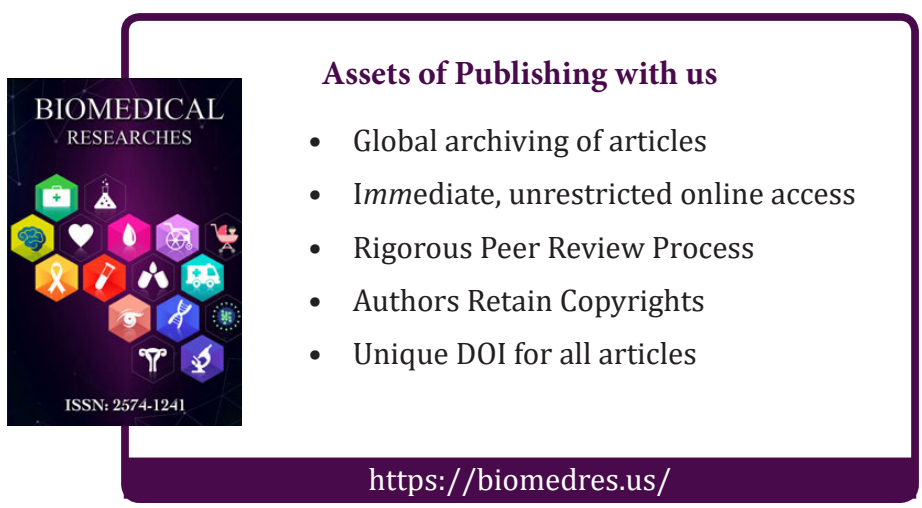

